\title{
Spondylodiscitis: A Retrospective Analysis of Clinical, Etiological, and Radiological Diagnosis
}

\author{
AFONSO CARDOSO, MD, LUÍS BARBOSA, MD, ANA MARTA COELHO, MD, JOÃO GONÇALVES \\ CORREIA, MD, HÉLDER LARGUINHO MAURÍCIO, MD, ÁLVARO LIMA, MD \\ Hospital Beatriz Ângelo, Loures, Portugal
}

\begin{abstract}
Background: Spondylodiscitis is an uncommon disease, and due to its indolent nature, it is often a late diagnosis. Great stress is put on the etiologic diagnosis, but blood cultures do not always yield positive results. Magnetic resonance imaging (MRI), despite being the diagnostic method of choice, is not always available. Our aim was to characterize the clinical presentation and to identify and check the efficacy of the etiologic and radiological methods of diagnosis of spondylodiscitis used at our hospital.

Methods: A retrospective study was conducted in which spontaneous spondylodiscitis cases were identified. The clinical presentation and the results of etiologic and radiological methods of diagnosis were analyzed.

Results: Over a period of 5 years, 34 patients fulfilled the inclusion criteria of the study. Regarding days of complaints, we identified a median of 9.5 days (1-547 days), with back pain being the predominant symptom. Fever was present in half the patients. Blood cultures were positive in 16 patients $(48.5 \%)$. Nineteen patients underwent a computed tomography (CT)-guided biopsy (positive in 7 patients [36.8\%]), and 10 patients underwent a surgical biopsy (positive in half of them). Overall, 27 patients (79.4\%) had an etiologic diagnosis. The diagnostic work-up consisted mostly of an initial CT scan followed by a confirmatory MRI. Of note, in 5 patients the CT scan did not reveal changes that were later confirmed by MRI. A total of 29 patients $(85.3 \%)$ underwent an MRI, with 28 being diagnostic.

Conclusions: Spondylodiscitis remain a difficult diagnosis. Blood cultures should always be obtained before antibiotic administration and a CT-guided or surgical biopsy should be done if needed. Our results confirm the importance of MRI as the imaging modality of choice and highlight the possibility of false-negative CT scans and the inability of CT to allow for a definitive diagnosis.
\end{abstract}

Other \& Special Categories

Keywords: spondylodiscitis, diagnosis, magnetic resonance imaging, computed tomography scan, CT-guided biopsy, surgical biopsy

\section{INTRODUCTION}

Spondylodiscitis refers to infection of the intervertebral disc and osteomyelitis of adjacent vertebral plates. ${ }^{1}$ This entity may sometimes have a devastating result.

With regard to epidemiology, this is an uncommon disease in developed countries. ${ }^{2}$ The incidence ranges from 0.2 per 100000 to 2.4 per 100000 person-years, a number that has been increasing in recent years. ${ }^{2,3}$ In developing countries, it is estimated that this rate is considerably higher. ${ }^{4}$ Among the identified risk factors, diabetes mellitus and advanced age stand out. ${ }^{5,6}$ Men are affected about 3 times more than women, and patients are also more affected between the sixth and eighth decades. ${ }^{6,7}$
This infection may have several causes, the most frequent being the hematogenous dissemination of infectious agents. ${ }^{8}$ Several organisms have been identified as responsible, with the most prevalent being Staphylococcus aureus. ${ }^{3,5,8}$ Clinical presentation is variable and symptoms and signs include spinal pain, fever, nausea, and weight loss. ${ }^{6}$ Due to the frequency of the entity, its indolent character, and the difficult differential diagnosis, the diagnosis is often delayed. ${ }^{6}$ This sometimes causes serious complications such as irreversible neurological deficits or death.

For the etiological diagnosis, blood cultures are often the first step to try to identify the causative agent. $^{5,8,9}$ An image-guided aspiration biopsy is advised in patients in whom the microbiologic diagnosis has not been established by blood cultures or serologic tests. 
Various radiological diagnostic methods are available to diagnose spondylodiscitis, with magnetic resonance imaging (MRI) being the method of choice. ${ }^{9}$ When MRI cannot be obtained, a combination of gallium and Tc99 scintigraphy, a computed tomography scan (CT), or a positron emission tomography is indicated. ${ }^{9}$

The main objective of this study was to understand how the etiologic and radiological diagnosis was achieved, the efficacy of the methods used, and to characterize the population that was admitted with this diagnosis to our hospital.

\section{METHODS}

Cases of spontaneous spondylodiscitis treated at our hospital from 2012 to 2016 were identified. This hospital is a secondary center that covers a population of about 278000 people. The hospital's database (WebGDH) was used for the search, from July 22-24, 2017, for the diagnoses of spondylodiscitis, psoas abscess, and tuberculosis of the spine (codes 7209/72081, 56731 and 01500-01506, respectively). The inclusion criteria for the study was a proven diagnosis of spondylodiscitis, based on a combination of clinical and laboratorial, confirmed by radiological features. Exclusion criteria were an unconfirmed diagnosis of spondylodiscitis, recent spine surgery ( $<3$ months) and treatment performed predominantly in another hospital.

A retrospective study was conducted. Data were collected by consulting patients' electronic files. The data of interest were patient demographics, personal history, comorbidities, hospitalization time, duration of complaints, admissions to the emergency department, clinical presentation, site of complaint, laboratory parameters, diagnostic work-up, and etiological studies. Because tuberculosis is more difficult to diagnose and it usually presents with a more insidious onset, we analyzed these cases as a subset, according to the collected data. Cases were classified as proposed by Pola et al. ${ }^{10}$ The study was approved by the institutional ethics committee.

Regarding the statistical analysis, data normality was tested using the Kolmogorov-Smirnov test. Continuous variables were presented as the median or mean. To analyze them, the Student $t$ test or the Mann-Whitney $U$ test was used, according to the data distribution. The proportions were expressed for nominal variables. To compare them we used the Pearson $\chi^{2}$ test or the Fisher exact test, according to the data distribution. A $P$ value less than .05 was
Table 1. Demographic and clinical data.

\begin{tabular}{lc}
\hline Parameter & Value \\
\hline Sex, n $(\%)$, male/female & $26(76.6) / 8(23.5)$ \\
Age, mean (range), y & $65.3(25-86)$ \\
Days of complaints, median (range) & $9.5(1-547)$ \\
Admissions to ED, mean (range) & $1.7(1-4)$ \\
Fever & $17(50)$ \\
$\quad$ n $(\%)$ & 38.3 \\
Mean, ${ }^{\circ} \mathrm{C}$ & $27(87.1)$ \\
Spinal pain at presentation, n $(\%)^{\mathrm{a}}$ & $4(11.8)$ \\
Motor deficit, n $(\%)$ & $6(21.4)$ \\
Sensory deficit, n $(\%)$ & $580(5900-29300)$ \\
Leukocytes & $33(97)$ \\
High CPR & $14.5(1-37)$ \\
$\quad$ n $(\%)$ & \\
$\quad$ Mean (range), mg/dL & $10(100)$ \\
High ESR & $79.3(56-140)$ \\
$\quad$ n $(\%)$ & $40.91(1-125)$ \\
$\quad$ Mean (range), mm/h & \\
Hospitalization days, mean (range) & \\
\hline
\end{tabular}

Abbreviations: CPR, C-reactive protein; ED, emergency department; ESR, erythrocyte sedimentation rate.

${ }^{a}$ All patients developed spinal pain during hospitalization.

${ }^{b}$ Data available in 10 patients.

considered significant. The statistical analysis was done with SPSS, version 20 (IBM Corp, Armonk, NY).

\section{RESULTS}

The search returned a total of 49 patients, and, after their files were consulted, 39 patients with a diagnosis of spondylodiscitis were identified (10 patients with other diagnoses or under study in another hospital were excluded). Of the 39 patients diagnosed with spondylodiscitis, 3 had undergone surgery on the spine (all in the month prior to the admission for spondylodiscitis) and therefore were excluded from the study. Two patients were treated predominantly in another hospital, for which they were also excluded.

Demographic and clinical data are presented in Table 1. Of note, among patients with a subsequent etiologic diagnosis of Mycobacterium tuberculosis, duration of complaints was significantly higher than in those with a diagnosis of another agent (175.6 versus 21.9 days, respectively; $P=.003$ ). Regarding the analytical evaluation, at admission, patients with a diagnosis of $M$. tuberculosis had a lower Creactive protein (CRP) level than other patients (7.6 versus 18.9 , respectively; $P=.008)$. Seventeen patients $(50 \%)$ had leukocytosis $(>10000)$, and in that population the mean value was 15911 . Fifteen patients $(44.1 \%)$ had an identified infectious source other than the spine, detailed in Table 2. Six patients $(17.6 \%)$ presented with septicemia. 
Table 2. Infectious sites at admission.

\begin{tabular}{lcc}
\hline Concomitant Infectious Sites & n & Percentage $^{\mathbf{a}}$ \\
\hline Urinary & 6 & 40 \\
Pulmonary & 4 & 26.7 \\
Meningitis & 2 & 13.3 \\
Dialysis access & 1 & 6.7 \\
Aortic vascular prosthesis & 1 & 6.7 \\
Gallbladder & 1 & 6.7 \\
\hline
\end{tabular}

${ }^{\mathrm{a}}$ The percentage refers to the total number of infectious sites.

The topography of the infection is present in Figure 1. Patients with lumbar involvement had mainly a nontuberculosis diagnosis $(85.7 \%)$, whereas patients with tuberculosis had mainly thoracic involvement $(75.0 \%)$. Patients with tuberculosis also showed a higher presence of bone destruction $(87.5 \%$ versus $31.6 \%)$.

Concerning the comorbidities of the patients in our group, 2 patients $(5.9 \%)$ had a history of noninstrumented spine surgery more than 4 years before the index event. Regarding medical comorbidities, diabetes mellitus was preponderant (12 patients, $35.3 \%)$. Ten patients $(29.4 \%)$ had more than 1 risk factor, and 11 patients $(32.4 \%)$ had a history of recent infection, mostly pulmonary.

Several cultural exams (blood cultures and CTguided or surgical biopsy; Figure 2) were performed and their results are summarized in Table 3. Blood cultures were drawn before antibiotic treatment in at least 25 patients $(75.8 \%)$. The etiological diagnoses are detailed in Table 4. Surgeries consisted mainly of posterior fixation and arthrodesis in cases of instability and in decompression and surgical debridement where neurologic compromise was present without other signs of instability.

In most cases, the diagnostic work-up consisted of an initial CT scan followed by a confirmatory MRI (average $=7.8$ days later [range, $0-15$ days]). Thirty patients $(88.2 \%)$ underwent a CT scan: 3

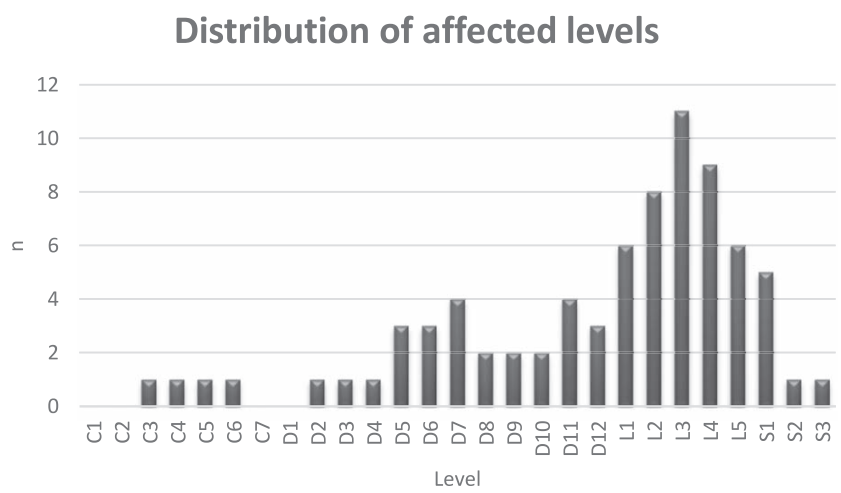

Figure 1. Topography of infections. The lumbar spine was the most affected $(50 \%)$, followed by the thoracic $(38.2 \%)$ and then the cervical spine $(11.8 \%)$.

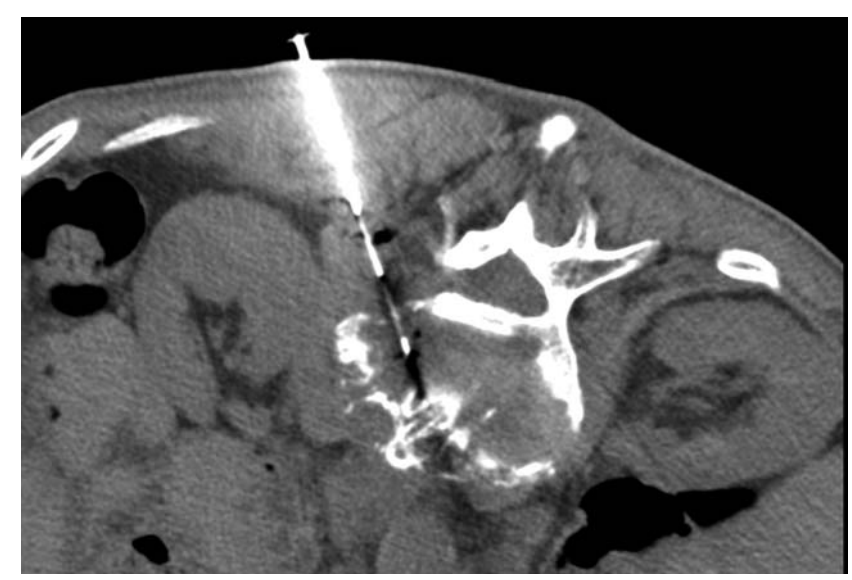

Figure 2. CT-guided vertebral biopsy. CT, computed tomography.

were diagnostic, 5 were negative, and in 22 , a diagnostic suspicion was raised (Figure 3). Twentynine patients $(85.3 \%)$ underwent an MRI; 28 were diagnostic and 1 was suspicious. Three patients $(8.8 \%)$ underwent a gallium scintigraphy; 2 were diagnostic and 1 was suspicious. One patient $(2.9 \%)$, unable to undergo MRI, was diagnosed by a combination of imaging, cultural, and clinical methods. Also noted were the presence of concomitant abscess of the psoas muscle in 12 patients $(35.3 \%)$, paravertebral abscesses in 14 patients $(41.2 \%)$, and epidural abscesses in 12 patients $(35.3 \%)$. Table 5 shows patient distribution according to the classification proposed by Pola et al. ${ }^{10}$

\section{DISCUSSION}

Spondylodiscitis has been described for more than a century, and although it remains a rare disease, it is the main manifestation of hematogenous osteomyelitis in patients over 50 years of age. ${ }^{11}$ In the time period we studied, 34 cases of spontaneous spondylodiscitis were identified. ${ }^{2,3}$

Our data show that this entity is more frequent in men in their seventh decade. Its indolent character was made evident by the long duration of symptoms before patients sought medical attention, with many

Table 3. Results of cultural exams. ${ }^{a}$

\begin{tabular}{lcccc}
\hline Test Result & $\begin{array}{c}\text { BC, } \\
\mathbf{n}(\%)\end{array}$ & $\begin{array}{c}\text { CT-Guided } \\
\text { VB, n (\%) }\end{array}$ & $\begin{array}{c}\text { Surgical-Guided } \\
\text { VB, n (\%) }\end{array}$ & $\begin{array}{c}\text { Total, } \\
\mathbf{n}(\%)\end{array}$ \\
\hline Positive & $16(48.5)$ & $7(36.8)$ & $5(50)$ & $27(79.4)$ \\
Negative & $17(51.5)$ & $12(63.2)$ & $5(50)$ & $7(20.6)$ \\
Total & $33(97.1)$ & $19(55.9)$ & $10(31.4)$ & $34(100)$ \\
\hline
\end{tabular}

Abbreviations: BC, blood culture; CT, computed tomography; VB, vertebral biopsy.

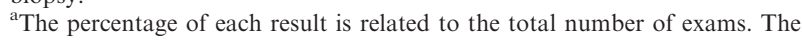
percentage of total exams refers to the total number of patients. 
Table 4. Description of etiological agents identified in cultural tests.

\begin{tabular}{lcc}
\hline Agent & $\begin{array}{c}\text { Study population, } \\
\mathbf{n}(\mathbf{\%})^{\mathrm{a}}\end{array}$ & $\begin{array}{c}\text { Surgical population, } \\
\mathbf{n} \mathbf{( \% )}\end{array}$ \\
\hline Staphylococcus aureus & $11(40.7)$ & $2(20.0)$ \\
$\quad$ MSSA & $5(45.5)$ & $2(100.0)$ \\
MRSA & $6(54.5)$ & $5(50.0)$ \\
Mycobacterium tuberculosis & $8(29.6)$ & $2(20.0)$ \\
Streptococcus spp & $4(14.8)$ & $1(10.0)$ \\
Klebsiella pneumoniae & $2(7.4)$ & \\
Staphylococcus epidermidis & $1(3.7)$ & $3(27.3)$ \\
Brucella melitensis & $1(3.7)$ & \\
No agent identified & $7(20.6)$ &
\end{tabular}

Abbreviations: MRSA, methicillin-resistant Staphylococcus aureus; MSSA, methicillin-sensitive $S$. aureus.

${ }^{\mathrm{a}}$ Within patients with identified agent.

patients not being diagnosed in the first emergency department attendance. Noteworthy also is the fact that half the patients did not present with fever and had only a modest rise in inflammatory parameters. However, there is a significant percentage of patients with concomitant infections (in accordance with the hematogenous spread route) and with other comorbidities. This pattern is also present in other series and confirms the reasons the diagnosis of this pathology is difficult and late. $5,6,12$

Objective neurological deficits have been identified in a relatively small number of patients, although these deficits are present in up to $33 \%$ of patients in some series. ${ }^{6}$ The best evidence in the literature suggests that this diagnosis should be suspected in patients with de novo spine pain or of recent aggravation accompanied by fever, pain accompanied by elevated CRP or erythrocyte sedimentation rate, or pain accompanied by infectious bacteremia or endocarditis. ${ }^{9}$ Homagk et al ${ }^{13}$ suggested a new diagnostic algorithm that combines pain, the value of CRP, and imaging features in MRI. The retrospective nature of our result made it impossible to study the application of this algorithm.

In terms of topography, it has been reported that spondylodiscitis mainly affects the lumbar spine, followed by the thoracic spine, and finally, the cervical spine $(58 \%, 30 \%$, and $11 \%$, respectively), as happened, roughly, in our patient group. 6,7 Cervical involvement is more frequently described in patients who use intravenous drugs (which we have not noticed), whereas dorsal involvement is a characteristic of tuberculosis, also present in this study. ${ }^{14}$ It should be noted that patients who had involvement of the cervical spine, had a concomitant diagnosis of meningitis and the agent identified was Streptococcus pneumoniae.
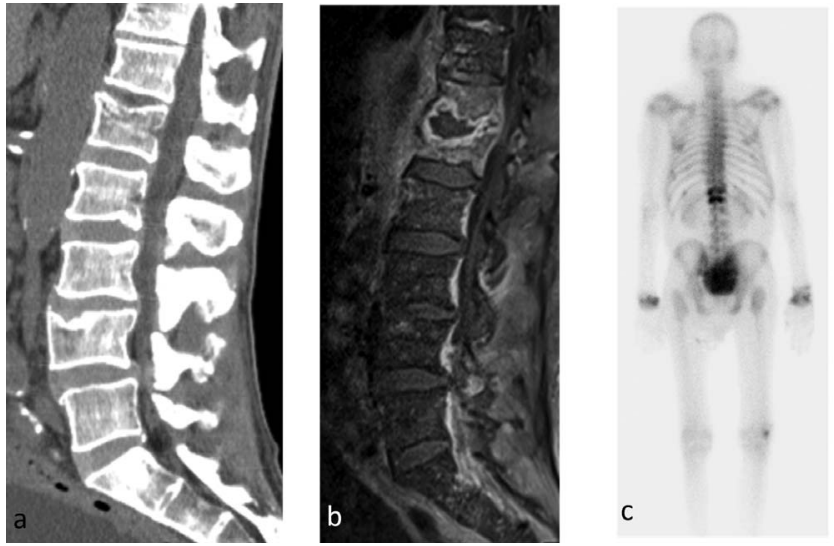

Figure 3. Imagiologic work-up of a case of spondylodiscitis. (a) CT scan showing recent somatic fracture of $\mathrm{L} 1$. (b) T2 MRI, done later, showing extensive structural and signal alteration, with edema and signal augmentation after gadolinium, consistent with spondylodiscitis. (c) Gallium scintigraphy showing hyperfixation consistent with spondylodiscitis. CT, computed tomography; MRI, magnetic resonance imaging.

Regarding cultural exams, their importance cannot be overestimated because they allow the etiological diagnosis and guide the antibiotic treatment, and, whenever possible, should precede the beginning of antibiotic therapy. ${ }^{9}$ It should be noted that in our study, as in the literature, blood cultures were positive in about $50 \%$ of patients. ${ }^{14}$ Image- or surgical-guided biopsy is indicated in the impossibility of identifying an agent through blood cultures or serological studies. ${ }^{9}$ However, regarding CTguided biopsy, we had positive results in $36.8 \%$ of patients, a lower yield rate than that descripted in the literature, a finding we attribute to the previous use of antibiotics. These were cases where blood cultures were mostly negative, and antibiotics were started before the biopsy. When possible, consideration should be given to postponing the start of antibiotic therapy until an etiologic diagnosis is

Table 5. Classification of spondylodiscitis according to the classification proposed by Pola et al. ${ }^{10}$

\begin{tabular}{crr}
\hline Classification & n & Percentage \\
\hline Type A & 13 & 38.2 \\
A.2 & 2 & 5.9 \\
A.3 & 6 & 17.7 \\
A.4 & 5 & 14.7 \\
Type B & 5 & 14.7 \\
B.1 & 2 & 5.9 \\
B. 2 & 2 & 5.9 \\
B.3 & 1 & 2.9 \\
Type C & 16 & 47.1 \\
C. 1 & 5 & 14.7 \\
C. 2 & 4 & 11.8 \\
C. 3 & 3 & 8.8 \\
C. 4 & 4 & 11.8 \\
\hline
\end{tabular}


made and not solely after harvesting blood cultures. It should be noted that the agent most commonly identified by methods other than blood culture was M. tuberculosis.

As for the agent of spondylodiscitis, Staphylococcus aureus was prominent, as in the literature. . $5,6,11$ However, it is important to note that the prevalence of methicillin-resistance is been increasingly identified. ${ }^{14}$ On a second note, other agents frequently implicated in the literature, such as Enterobacteriaceae and Staphylococcus coagulase-negative, did not have as much expression in our sample $(7 \%-33 \%$ versus $7.4 \%$ and $5 \%-16 \%$ versus $3.7 \%$, respectively). ${ }^{6}$

Concerning tuberculosis, it continues to be the main cause of spondylodiscitis worldwide, accounting for about $9 \%$ to $46 \%$ of cases in developing countries. ${ }^{4}$ In developed countries, this entity is associated with ethnic groups from endemic regions. ${ }^{14}$ This is probably the reason $M$. tuberculosis is the second most frequent agent in our series (and the most frequent in the surgical group), given that our hospital receives a lot of expatriated patients from such regions. This prevalence does not occur in all series of patients. ${ }^{6}$ Also notable are the indolent character of spondylodiscitis, hereby demonstrated by a much longer period of complaints, time to diagnosis, and a lower CRP level at presentation.

MRI is the imaging modality of choice, and in most cases, it confirms the diagnosis. ${ }^{6,9}$ It is necessary to keep the level of suspicion high, because, as happened with 5 of our patients, the CT scan may not reveal changes that are later confirmed by MRI. If it is impossible to perform an MRI, the examination of choice may be scintigraphy with a combination of gallium/Tc99, CT, or a proton emission tomography. ${ }^{9}$

As negative aspects of this study, it is necessary to point out its retrospective nature, which has limited, in some circumstances, the complete collection of data.

\section{CONCLUSIONS}

Given the potential complications of this diagnosis and its increased incidence, it is important to raise the clinical suspicion of spondylodiscitis in appropriate cases. Blood cultures should always be obtained before antibiotic administration and consideration should be given to postponing the start of antibiotics until the etiologic diagnosis is made.
When these are negative, a CT-guided or a surgical biopsy should be done. Our results confirm the importance of MRI as the imaging modality of choice and highlight the possibility of false negative CT scans and the inability of CT to allow for a definitive diagnosis. The antibiotic therapy should cover the most frequent agents, taking into account methicillin-resistant Staphylococcus aureus and local particularities, as a high prevalence of tuberculosis.

\section{REFERENCES}

1. Zarghooni K, Röllinghoff $\mathrm{M}$, Sobottke R, Eysel P. Treatment of spondylodiscitis. Int Orthop. 2012;36(2):405-411. doi:10.1007/s00264-011-1425-1

2. Cheung WY, Luk KDK. Pyogenic spondylitis. Int Orthop. 2012;36(2):397-404. doi:10.1007/s00264-011-1384-6

3. Acosta FL, Galvez LF, Aryan HE, Ames CP. Recent advances: infections of the spine. Curr Infect Dis Rep. 2006;8(5):390-393. doi:10.1007/s11908-006-0050-4

4. Tuli SM. Tuberculosis of the spine: a historical review. Clin Orthop Relat Res. 2007;460:29-38. doi:10.1097/BLO. 0b013e318065b75e

5. Carragee EJ. Pyogenic vertebral osteomyelitis. J Bone Joint Surg Am. 1997;79(6):874-880. http://www.ncbi.nlm.nih. gov/pubmed/9199385. Accessed March 17, 2018.

6. Mylona E, Samarkos M, Kakalou E, Fanourgiakis P, Skoutelis A. Pyogenic vertebral osteomyelitis: a systematic review of clinical characteristics. Semin Arthritis Rheum. 2009;39(1):10-17. doi:10.1016/j.semarthrit.2008.03.002

7. Malawski SK, Lukawski S. Pyogenic infection of the spine. Clin Orthop Relat Res. 1991;(272):58-66. http://www. ncbi.nlm.nih.gov/pubmed/1934752. Accessed March 17, 2018.

8. Zimmerli W. Vertebral osteomyelitis. $N$ Engl J Med. 2010;362(11):1022-1029. doi:10.1056/NEJMcp0910753

9. Berbari EF, Kanj SS, Kowalski TJ, et al. 2015 Infectious Diseases Society of America (IDSA) clinical practice guidelines for the diagnosis and treatment of native vertebral osteomyelitis in adults. Clin Infect Dis. 2015;61(6):e26-e46. doi:10.1093/cid/ civ482

10. Pola E, Autore G, Formica VM, et al. New classification for the treatment of pyogenic spondylodiscitis: validation study on a population of 250 patients with a follow-up of 2 years. Eur Spine J. 2017;26(S4):479-488. doi:10.1007/s00586-017-5043-5

11. Jensen AG, Espersen F, Skinhøj P, Rosdahl VT, Frimodt-Møller N. Increasing frequency of vertebral osteomyelitis following Staphylococcus aureus bacteraemia in Denmark 1980-1990. J Infect. 1997;34(2):113-118. doi:10.1016/S01634453(97)92395-1

12. Torda AJ, Gottlieb T, Bradbury R. Pyogenic vertebral osteomyelitis: analysis of 20 cases and review. Clin Infect Dis. 1995;20(2):320-328. doi:10.1093/clinids/20.2.320

13. Homagk L, Homagk N, Klauss JR, Roehl K, Hofmann GO, Marmelstein D. Spondylodiscitis severity code: scoring system for the classification and treatment of non-specific spondylodiscitis. Eur Spine J. 2016;25(4):1012-1020. doi:10. 1007/s00586-015-3936-8

14. Gouliouris T, Aliyu SH, Brown NM. Spondylodiscitis: 
update on diagnosis and management. J Antimicrob Chemother. 2010;65(suppl 3):iii11-iii24. doi:10.1093/jac/dkq303

Disclosures and COI: The authors received no funding for this study and declare no conflicts of interest.

Corresponding Author: Afonso Cardoso, Hospital Beatriz Angelo, Av Carlos Teixeira 514, 2674 Loures, Portugal. Phone: +351 219847209; Fax
+351219847209; Email: afonso.cardoso@ hbeatrizangelo.pt.

Published 30 April 2020

This manuscript is generously published free of charge by ISASS, the International Society for the Advancement of Spine Surgery. Copyright (C) 2020 ISASS. To see more or order reprints or permissions, see http://ijssurgery.com. 